\title{
Evaluation of probability of linkages between enterprises and farmer's potatoes in Lam Dong province
}

\author{
Nam H. Tran*, Vu Le, \& Lap D. Tran \\ Faculty of Economics, Nong Lam University, Ho Chi Minh City, Vietnam
}

\begin{abstract}
ARTICLE INFO
Research Paper

Received: July 09, 2018 Revised: September 27, 2018 Accepted: December 05, 2018

Keywords

Linkages

Multinomial logistic regression Potato production Probability

\section{${ }^{*}$ Corresponding author}

Tran Hoai Nam

Email: hoainam@hcmuaf.edu.vn
\end{abstract}

ABSTRACT

In this study, the collaboration between buyer and the famers in potato production was evaluated by using a multinomial Logistic regression model with MLE estimating. The data were collected by directly interviewing of 245 farmers at the Xuan Tho commune, Da Lat city and Don Duong district, Lam Dong province. Results of the research showed that a tight collaboration between the companies and farmers would increase in a higher productivity. The estimation showed that the probability of farmer which would and would not collaborate with buyer were $14.6 \%\left(\mathrm{Y}_{2} / \mathrm{Y}_{1}\right)$ and $63.0 \%\left(\mathrm{Y}_{3} / \mathrm{Y}_{1}\right)$. The factors affecting the proability of linkages between enterprises and farmers were enterprises and farmers were experience, farm, size, profit, policy supports and gender. The results also revealed that when price of potatoes increase, farmers would not comply with argreement.

Cited as: Tran, N. H., Le, V., \& Tran, L. D. (2019). Evaluation of probability of linkages between enterprises and farmer's potatoes in Lam Dong province. The Journal of Agriculture and Development 18(1), 1-8. 


\title{
Đánh giá khả năng tham gia liên kết giữa doanh nghiệp và nông hộ trong sản xuất khoai tây tại tỉnh Lâm Đồng
}

\author{
Trần Hoài Nam*, Lê Vũ \& Trần Độc Lập \\ Khoa Kinh Tế, Trường Đại Học Nông Lâm TP. Hồ Chí Minh, TP. Hồ Chí Minh
}

THÔNG TIN BÀI BÁO
Bài báo khoa học
Ngày nhận: 09/07/2018
Ngày chỉnh sửa: $27 / 09 / 2018$
Ngày chấp nhận: 05/12/2018
Từ khóa
Khả năng
Liên kết
Mô hình logit đa thức
Sản xuất khoai tây
*Tác giả liên hệ
Trần Hoài Nam
Email: hoainam@hcmuaf.edu.vn

\section{1. Đặt Vấn Đề}

Tỉnh Lâm Đồng được xem là một trong những vùng canh tác khoai tây trọng điểm của cả nước. Theo Lam Dong DARD (2018), diện tích trồng khoai tây hàng năm của tỉnh khoảng 1.500 - 1.600 ha, sản lượng 35.000 tấn/năm. Tuy nhiên, trước áp lực cạnh tranh của khoai tây Trung Quốc thì việc sản xuất và tiêu thụ khoai tây tại Lâm Đồng gặp nhiều khó khăn, diện tích trồng khoai tây đã giảm mạnh trong thời gian vừa qua. Trong bối cảnh đó, xây dựng mối liên kết giữa doanh nghiệp và nông hộ có vai trò ngày càng quan trọng. Liên kết trong sản xuất nông nghiệp đã được áp dụng rất thành công thông qua các tập đoàn kinh doanh nông nghiệp đa quốc gia ở một số nước như ở Mỹ, Thái Lan, Brazil, Chile, Mexico (Tran \& Ikuo, 2012).

Ở nước ta, Chính phủ cũng rất quan tâm đến tiêu thụ hàng hóa nông sản cho người nông dân thông qua hợp đồng. Chính phủ đã ban hành Quyết định số 80/2002/QĐ-TTg về chính sách khuyến khích tiêu thụ hàng hóa nông sản thông qua hợp đồng giữa nông dân và doanh nghiệp, nhưng đến nay việc tiêu thụ hàng hóa thông qua hợp đồng vẫn còn gặp nhiều khó khăn (MARD, 2008). Thực tế cũng có nhiều doanh nghiệp ký hợp đồng cung ứng vật tư nông nghiệp và bao tiêu nông sản hàng hóa cho nông dân nhưng nhiều hợp đồng không thực hiện được. Tùy từng trường hợp cụ thể về biến động giá cả, hợp đồng bị phá vỡ hoặc từ phía doanh nghiệp, hoặc từ phía nông dân (Tran \& Pham, 2014). Do đó, xây dựng và phát triển các mối liên kết gắn sản xuất và tiêu thụ sản phẩm nông nghiệp là chủ trương đúng đắn nhằm giúp việc sản xuất tập trung, nâng cao thu nhập và ổn định đời sống của nông hộ. Vì vậy, nghiên cứu này được tiến hành với mục tiêu 
là phân tích các yếu tố ảnh hưởng đến khả năng tham gia liên kết giữa doanh nghiệp và nông hộ trong sản xuất khoai tây, từ đó gợi ý một số giải pháp nhằm nâng cao hiệu quả liên kết trong sản xuất khoai tây.

\section{Cơ Sở Lý Luận và Phương Pháp Nghiên Cứu}

\subsection{Tổng quan tài liệu}

Một trong các mục tiêu của liên kết nhằm phân bổ lợi ích và rủi ro để các tác nhân tham gia cùng nhau hưởng lợi từ sự liên kết này. Trong ngành nông nghiệp, liên kết thường được phân chia thành liên kết dọc và liên kết ngang. Liên kết dọc là liên kết giữa các tác nhân theo đường đi của sản phẩm từ người sản xuất đến người tiêu dùng, trong khi liên kết ngang là liên kết các tác nhân, các đối tượng cùng tham gia vào các hoạt động tương tự nhau như liên kết các hộ nông dân với nhau, các hợp tác xã (Tu, 2016). Đã có nhiều nghiên cứu về liên kết dọc trong sản xuất nông sản theo hợp đồng ở nước ta, các tác giả đã chỉ ra khả năng liên kết sản xuất theo hợp đồng của nông hộ chịu ảnh hưởng tích cực từ các yếu tố như trình độ học vấn, tuổi chủ hộ, diện tích, điều kiện hạ tầng giao thông, nhóm dân tộc và quy mô sản xuất (Do \& Tran, 2013; Nguyen \& La, 2014; Nguyen \& ctv., 2017). Tuy nhiên, quá trình hình thành và phát triển những liên kết đang gặp rất nhiều khó khăn, các liên kết thiếu sự chặt chẽ giữa các bên, đồng thời lợi ích do hợp đồng mang lại chưa đủ hấp dẫn nên mối liên kết này rất dễ bị phá vỡ (Tran \& Ikuo, 2012; Phung \& Pham 2014; Tran \& ctv., 2016). Tuy nhiên, việc xem xét mức giá bán và qui mô sản xuất ảnh hưởng như thế nào đến khả năng liên kết của hộ sản xuất vẫn còn ít. Nghiên cứu này, sẽ xem xét ảnh hưởng của các yếu tố giá cả và quy mô sản xuất đến mối liên kết giữa doanh nghiệp và nông hộ trong sản xuất khoai tây.

\subsection{Nguồn số liệu}

Số liệu được thu thập từ 245 nông hộ canh tác khoai tây (9/2017) tại xã Xuân Thọ, thành phố Đà Lạt và huyện Đơn Dương, tỉnh Lâm Đồng. Đây là khu vực tập trung sản xuất khoai tây lớn nhất, trong đó nông hộ sản xuất khoai tây tại xã Xuân Thọ không có tham gia liên kết cũng như sẽ tham gia liên kết và nông hộ tại huyện Đơn Dương đã tham gia liên kết. Số liệu được thu thập thông qua phỏng vấn trực tiếp bằng bảng câu hỏi. Ngoài ra, còn thu thập các thông tin thứ cấp từ nhiều nguồn khác nhau, bao gồm các tài liệu, các báo cáo, các nghiên cứu trong và ngoài nước được thu thập qua các nguồn khác nhau để phục vụ cho nghiên cứu. Các thông tin đã thu thập được tổng hợp, tính toán và phân tích bằng phần mềm Excel và Limdep 9.0.

\subsection{Phương pháp phân tích và xử lý số liệu}

Trong nghiên cứu này, phương pháp hồi quy logit đa thức được sử dụng để xác định tác động của các yếu tố đến khả năng tham gia liên kết giữa doanh nghiệp và nông hộ trong sản xuất khoai tây. Mô hình hồi quy logit đa thức được sử dụng nhằm dự đoán và giải thích mối quan hệ của các biến trong nhiều lĩnh vực khác nhau như kinh doanh, kinh tế, giáo dục, chăm sóc sức khoẻ, cũng như trong lĩnh vực nông nghiệp. Mô hình hồi quy logit đa thức tương tự như mô hình hồi quy logit nhị thức nhưng biến phụ thuộc là biến định tính có nhiều hơn 2 trạng thái (Pannapa \& Dennis, 2015).

Mô hình hồi quy đa thức được thể hiện như sau:

$$
\log \left(\frac{\mathrm{p}_{\mathrm{ij}}}{\mathrm{p}_{\mathrm{i} 1}}\right)=x_{\mathrm{i}} \beta_{\mathrm{j}} ; \mathrm{j}=1, \ldots, \mathrm{j} ; \mathrm{i}=1, \ldots, \mathrm{N}
$$

Trong đó: $\mathrm{p}_{\mathrm{ij}}$ là xác suất của $\left(\mathrm{Y}=\mathrm{j} / \mathrm{x}_{\mathrm{i}}\right)$ :

$$
\mathrm{p}\left(\mathrm{y}=\mathrm{j} / x_{\mathrm{i}}\right)=\frac{\exp \left(x_{\mathrm{i} \beta_{\mathrm{j}}}\right)}{1+\sum_{\mathrm{j}=1}^{\mathrm{j}} \exp \left(x_{\mathrm{i} \beta_{\mathrm{j}}}\right)}
$$

Các hệ số hồi qui sẽ được ước lượng bằng phương pháp ước lượng hợp lý cực đại (Maximum Likelihood Estimation). Giá trị $\mathrm{p}_{\mathrm{i}}$ xác suất nông hộ thứ i tham gia liên kết trong sản xuất khoai tây $(\mathrm{p}=1$ : nếu hộ không tham gia; $\mathrm{p}=2$ : nếu hộ sẽ tham gia; $\mathrm{p}=3$ : nếu hộ đã tham gia), nên mô hình được viết lại:

$$
\begin{aligned}
& \mathrm{p}(\mathrm{Y}=1)=\frac{1}{1+\sum_{\mathrm{j}=1}^{\mathrm{j}} \exp \left(x_{\mathrm{i} \beta_{\mathrm{j}}}\right)} \\
& \mathrm{p}(\mathrm{Y}=\mathrm{j})=\frac{\exp \left(x_{\mathrm{i} \beta_{\mathrm{j}}}\right)}{1+\sum_{\mathrm{j}=1}^{\mathrm{j}} \exp \left(x_{\mathrm{i} \beta_{\mathrm{j}}}\right)}
\end{aligned}
$$

$\mathrm{X}_{\mathrm{i}}$ là biến độc lập với $\mathrm{X}_{1}$ tuổi chủ hộ (năm); $\mathrm{X}_{2}$ trình độ học vấn của chủ hộ (năm); $\mathrm{X}_{3}$ kinh nghiệm sản xuất nông nghiệp của hộ (năm); $\mathrm{X}_{4}$ diện tích (ha); $\mathrm{X}_{5}$ lợi nhuận (triệu đồng/ha); $\mathrm{X}_{6}$ 
chính sách hỗ trợ (sử dụng thang đo Likert và tổng hợp từ các biến như tập huấn khuyến nông; hỗ trợ thông tin thị trường và hỗ trợ vốn); $\mathrm{D}_{1}$ giới tính chủ hộ (1: nam, 0: nữ).

\section{Kết Quả và Thảo Luận}

\section{1. Đặc điểm của hộ điều tra}

Nghiên cứu tiến hành phỏng vấn 62 hộ sản xuất khoai tây không tham gia, 61 hộ sẽ tham gia và 122 hộ đã tham gia liên kết. Kết quả thống kê từ Bảng 1 cho thấy đối tượng khảo sát khá đa dạng và phong phú về tuổi tác cũng như trình độ học vấn. Độ tuổi trung bình của chủ hộ vào khoảng 46 tuổi (trong đó mức tuổi từ 40 đến 50 tuổi chiếm tỷ trọng cao nhất 29,03\% (hộ không liên kết), $26,23 \%$ (hộ sẽ tham gia liên kết) và $34,43 \%$ (hộ đã tham gia) ở độ tuổi này nông hộ vẫn còn đủ sức khoẻ để trực tiếp tham gia sản xuất.

Đồng thời, trình độ học vấn của nông hộ chủ yếu là trung học cơ sở và trung học phổ thông, điều này tạo nhiều thuận lợi cho việc nắm bắt thông tin thị trường cũng như tiếp cận khoa học kỹ thuật khi thực hiện liên kết. Bên cạnh đó, kinh nghiệm là một trong những yếu tố có ảnh hưởng nhất định đến sản xuất. Dựa vào kết quả thống kê cho thấy, kinh nghiệm trong sản xuất của nông hộ trên 20 năm chiếm 32,26\% (hộ không tham gia), $42,62 \%$ (hộ sẽ tham gia) và $15,57 \%$ (hộ đã tham gia) với quy mô sản xuất chủ yếu ở mức $1.000-5.000 \mathrm{~m}^{2}$.

\subsection{So sánh hiệu quả tài chính trong sản xuất khoai tây}

Theo kết quả tính toán được thể hiện ở Bảng 2 cho thấy, chi phí sản xuất khoai tây của nông hộ trung bình là 13,653 triệu đồng/1000 $\mathrm{m}^{2}$ và những hộ tham gia liên kết có chí phí sản xuất nhỏ hơn các hộ không tham gia liên kết. Trong tất cả các chi phí, chi phí phân bón chiếm tỷ trọng cao nhất trong cơ cấu chi phí sản xuất. Doanh thu trung bình của nông hộ là 33,836 triệu đồng/1000 $\mathrm{m}^{2}$. Theo đó, hiệu quả của nông hộ trong sản xuất khoai tây theo hướng liên kết cao hơn so với nông hộ không tham gia liên kết (lợi nhuận/chi phí của hộ liên kết là 1,7 lần, của hộ sẽ tham gia là 1,4 lần và hộ không tham gia là 1,2 lần). Điều này phần nào lý giải tầm quan trọng trong mối liên kết sản xuất khoai tây như hiện nay.

\subsection{Phân tích các yếu tố ảnh hưởng đến khả năng tham gia liên kết giữa doanh nghiệp và nông hộ trong sản xuất khoai tây}

\subsubsection{Thực trạng liên kết trong sản xuất khoai tây của nông hộ}

Theo Lam Dong DARD (2018), diện tích trồng khoai tây tại Lâm Đồng chủ yếu tập trung tại TP. Đà Lạt (607 ha), Đơn Dương (464 ha), Lạc Dương (50 ha), Đức Trọng (28 ha) và huyện Lâm Hà (6 ha). Trong đó, sản lượng khoai tây sản xuất có hợp đồng tiêu thụ chiếm khoảng 35 - 40\% (chủ yếu nông hộ ký hợp đồng tiêu thụ với công ty PepsiCo VN và Công ty Orion) và còn khoảng 60 - $65 \%$ sản lượng khoai tây chưa có hợp đồng tiêu thụ (nông hộ chủ yếu bán cho tư thương khắp nơi với giá cả không ổn định).

Khi nông hộ tham gia hợp đồng liên kết sẽ được công ty hỗ trợ vật tư đầu vào và kỹ thuật sản xuất. Tuy nhiên, giá bán sản phẩm được quy định trong hợp đồng là cố định, do đó khi giá bán có biến động thì việc xác định giá sản phẩm rất khó khăn, dẫn đến tình trạng doanh nghiệp và nông hộ chưa gắn kết với nhau, cả doanh nghiệp và nông hộ đều vi phạm các điều khoản cam kết, dẫn đến hợp đồng dễ bị phá vỡ (trong vụ 2017 số hộ vi phạm hợp đồng là 6 hộ chiếm 4,91\% số hộ tham gia hợp đồng).

\subsubsection{Lợi ích khi tham gia liên kết của nông hộ}

Để tạo điều kiện cho các hộ trồng khoai tây hoàn thành hợp đồng đã ký kết, công ty Pepsi, Orion đã triển khai nhiều biện pháp nhằm hỗ trợ sản xuất. Bảng 3 thể hiện các lợi ích khác nhau mà nông hộ có thể nhận được. Kết quả cho thấy, $100 \%$ hộ khi tham gia liên kết có lợi ích cụ thể là được hỗ trợ vật tư nông nghiệp (đặc biệt là giống khoai tây) và tham dự các lớp tập huấn về kỹ thuật, 75,4\% nâng cao thu nhập. Các nông hộ cho rằng họ được trao đổi kinh nghiệm sản xuất với nhau cũng là một lợi ích quan trọng mà mình nhận được khi tham gia liên kết $(68,03 \%)$. Qua đây cho thấy rằng lợi ích cụ thể và thiết thực nhất đối với nông hộ khi tham gia liên kêt là được nâng cao trình độ khoa học kỹ thuật, trao đổi kinh nghiệm trong sản xuất và được hỗ trợ vật tư nông nghiệp. 
Bảng 1. Thông tin chung về đối tượng phỏng vấn

\begin{tabular}{|c|c|c|c|c|c|c|}
\hline \multirow{2}{*}{ Chỉ tiêu } & \multicolumn{2}{|c|}{ Hộ không tham gia } & \multicolumn{2}{|c|}{ Hộ sẽ tham gia } & \multicolumn{2}{|c|}{ Hộ tham gia } \\
\hline & $\begin{array}{c}\text { Tần số } \\
\text { (hộ) }\end{array}$ & $\begin{array}{c}\text { Tỷ trọng } \\
(\%)\end{array}$ & $\begin{array}{c}\text { Tần số } \\
\text { (hộ) }\end{array}$ & $\begin{array}{c}\text { Tỷ trọng } \\
(\%)\end{array}$ & $\begin{array}{c}\text { Tần số } \\
\text { (hộ) }\end{array}$ & $\begin{array}{c}\text { Tỷ trọng } \\
(\%)\end{array}$ \\
\hline \multicolumn{7}{|l|}{ Giới tính chủ hộ } \\
\hline Nam & 43 & 69,35 & 35 & 57,38 & 79 & 64,75 \\
\hline Nũ & 19 & 30,65 & 26 & 42,62 & 43 & 35,25 \\
\hline \multicolumn{7}{|l|}{ Tuổi chủ hộ } \\
\hline$\leq 30$ tuổi & 5 & 8,06 & 7 & 11,48 & 23 & 18,84 \\
\hline 30 tuổi - 40 tuổi & 16 & 25,81 & 11 & 18,03 & 36 & 29,51 \\
\hline 40 tuổi - 50 tuổi & 18 & 29,03 & 16 & 26,23 & 42 & 34,43 \\
\hline 50 tuổi - 60 tuổi & 14 & 22,58 & 12 & 19,67 & 13 & 10,66 \\
\hline$>60$ tuổi & 9 & 14,52 & 15 & 24,59 & 8 & 6,56 \\
\hline \multicolumn{7}{|l|}{ Trình độ học vấn } \\
\hline Mù chữ & 0 & 0,00 & 0 & 0,00 & 0 & 0,00 \\
\hline Tiểu học & 9 & 14,52 & 9 & 14,75 & 1 & 0,82 \\
\hline Trung học cơ sở & 30 & 48,39 & 29 & 47,54 & 10 & 8,20 \\
\hline Trung học phổ thông & 22 & 35,48 & 23 & 37,70 & 53 & 43,44 \\
\hline Cao đẳng - Đại học & 1 & 1,61 & 0 & 0,00 & 58 & 47,54 \\
\hline \multicolumn{7}{|l|}{ Kinh nghiệm } \\
\hline$\leq 5$ năm & 4 & 6,45 & 6 & 9,84 & 9 & 7,38 \\
\hline 5 năm - 10 năm & 15 & 24,19 & 11 & 18,03 & 25 & 20,49 \\
\hline 10 năm - 15 năm & 6 & 9,68 & 6 & 9,84 & 36 & 29,52 \\
\hline 15 năm - 20 năm & 17 & 27,42 & 12 & 19,67 & 32 & 26,24 \\
\hline > 20 năm & 20 & 32,26 & 26 & 42,62 & 19 & 15,57 \\
\hline \multicolumn{7}{|l|}{ Qui mô sản xuất } \\
\hline$\leq 1.000 \mathrm{~m}^{2}$ & 1 & 1,61 & 3 & 4,92 & 6 & 4,92 \\
\hline $1.000 \mathrm{~m}^{2}-5.000 \mathrm{~m}^{2}$ & 33 & 53,23 & 29 & 47,54 & 33 & 27,05 \\
\hline $5.000 \mathrm{~m}^{2}-10.000 \mathrm{~m}^{2}$ & 15 & 24,19 & 19 & 31,15 & 45 & 36,88 \\
\hline$>10.000 \mathrm{~m}^{2}$ & 13 & 20,97 & 10 & 16,39 & 38 & 31,15 \\
\hline
\end{tabular}

3.3.3. Mô hình hồi quy các yếu tố ảnh hưởng đến khả năng tham gia liên kết trong sản xuất khoai tây

Kết quả hồi quy trong mô hình Logit đa thức được thể hiện trong Bảng 4. Hệ số $\mathrm{R}^{2}$ của mô hình là $27,5 \%$ và $\mathrm{P}(\mathrm{F}$-stat $)=0,000$ nhỏ hơn rất nhiều so với mức $\alpha=5 \%$, điều này cho thấy sự phù hợp của mô hình hồi quy logit đa thức và các biến độc lập trong mô hình giải thích được $27,5 \%$ cho quyết định tham gia liên kết sản xuất khoai tây của nông hộ. Xác suất hộ sẽ tham gia liên kết là 14,6\% $\left(\mathrm{Y}_{2} / \mathrm{Y}_{1}\right)$ và hộ tham gia liên kết là $63,0 \%\left(\mathrm{Y}_{3} / \mathrm{Y}_{1}\right)$.

Kết quả hồi quy từ Bảng 4 cho thấy, các biến như kinh nghiệm, diện tích đất nông nghiệp, lợi nhuận, chính sách hỗ trợ và giới tính có ảnh hưởng đến quyết định tham gia liên kết của nông hộ. Trong khi đó, các yếu tố về đặc điểm nhân khẩu học như tuổi chủ hộ, trình độ học vấn đều không có ý nghĩa thống kê.

Kết quả trình bày trong Bảng 5 thể hiện tác động biên của các yếu tố đến hệ số odds tương đối (relative odds ration) của lựa chọn sẽ tham gia liên kết, tham gia liên kết với kết cục cơ sở (nông hộ không tham gia liên kết được chọn là kết cục cơ sở). Hệ số hồi quy của một yếu tố càng cao chứng tỏ tác động biên của yếu tố đó đến hệ số odds tương đối càng lớn, tức yếu tố đó tác động càng mạnh đến khả năng tham gia liên kết. Trong mô hình này, khi kinh nghiệm của nông hộ tăng thêm một năm thì khả năng tăng tham gia liên kết tăng lên 3\% (hộ sẽ tham gia) và $4,1 \%$ (hộ tham gia); qui mô sản xuất của hộ tăng thêm 1 ha thì khả năng tăng tham gia liên kết tăng lên 19,6\% (hộ sẽ tham gia) và 18,3\% (hộ tham gia); tương tự khi chính sách hỗ trợ tăng thêm một điểm thì sẽ tăng khả năng tham gia liên kết $8,2 \%$ và $19,5 \%$. Tuy nhiên, khi lợi nhuận tăng thêm 1 triệu đồng/ha thì khả năng tham gia 
Bảng 2. So sánh hiệu quả trong sản xuất khoai tây

\begin{tabular}{lcccc}
\hline \multirow{2}{*}{ Chỉ tiêu } & ĐVT & $\begin{array}{c}\text { Hộ không } \\
\text { tham gia }\end{array}$ & $\begin{array}{c}\text { Hộ sẽ } \\
\text { tham gia }\end{array}$ & Hộ tham gia \\
\hline Chi phí sản xuất & $1000 \mathrm{~d} / 1000 \mathrm{~m}^{2}$ & 14.953 & 14.250 & 11.968 \\
Chi phí vật chất & $1000 \mathrm{~d} / 1000 \mathrm{~m}^{2}$ & 9.439 & 9.985 & 8.533 \\
Chi phí giống & $1000 \mathrm{~d} / 1000 \mathrm{~m}^{2}$ & 2.033 & 2.033 & 2.033 \\
Chi phí phân bón & $1000 \mathrm{~d} / 1000 \mathrm{~m}^{2}$ & 5.299 & 5.140 & 3.650 \\
Chi phí thuốc BVTV & $1000 \mathrm{~d} / 1000 \mathrm{~m}^{2}$ & 2.108 & 1.850 & 1.250 \\
Khấu hao & $1000 \mathrm{~d} / 1000 \mathrm{~m}^{2}$ & 723 & 962 & 1.600 \\
Chi phí lao động & $1000 \mathrm{~d} / 1000 \mathrm{~m}^{2}$ & 4.791 & 4.265 & 3.435 \\
Chi phí lao động nhà & $1000 \mathrm{~d} / 1000 \mathrm{~m}^{2}$ & 2.092 & 1.865 & 1.865 \\
Chi phí lao động thuê & $1000 \mathrm{~d} / 1000 \mathrm{~m}^{2}$ & 2.699 & 2.400 & 1.570 \\
& & & & \\
Kết quả sản xuất & $1000 \mathrm{~d}$ & 33.415 & 34.280 & 32.450 \\
Doanh thu & $1000 \mathrm{~d}$ & 18.462 & 20.030 & 20.482 \\
Lợi nhuận & $1000 \mathrm{~d}$ & 20.554 & 21.895 & 22.347 \\
Thu nhập & & & & \\
Hiệu quả kinh tế & Lần & 1,2 & 1,4 & 1,7 \\
Lợi nhuận/chi phí & Lần & 1,4 & 1,5 & 1,9 \\
Thu nhập/chi phí & Lần & 2,2 & 2,4 & 2,7 \\
Doanh thu/chi phí & & &
\end{tabular}

Bảng 3. Các lợi ích khi tham gia liên kết

\begin{tabular}{lcc}
\hline $\begin{array}{l}\text { Các lợi ích khi tham gia } \\
\text { liên kết }\end{array}$ & $\begin{array}{c}\text { Tần số } \\
(\text { hộ})\end{array}$ & $\begin{array}{c}\text { Tỷ lệ } \\
(\%)\end{array}$ \\
\hline Tăng thu nhập & 92 & 75,40 \\
Được hỗ trợ vật tư NN & 122 & 100,00 \\
Được trao đổi kinh nghiệm & 83 & 68,03 \\
Được tập huấn KHKT & 122 & 100,00 \\
Được hỗ trợ vốn & 24 & 19,67 \\
\hline
\end{tabular}

liên kết giảm 1,4\% (hộ sẽ tham gia) và $0,5 \%$ (hộ đã tham gia). Đề cập đến khả năng phá vỡ liên kết giữa doanh nghiệp và nông hộ, Tran \& Ikuo (2012) cho rằng, nông hộ ở nước ta hay phá vỡ hợp đồng đã ký kết với các doanh nghiệp do khả năng chịu đựng rủi ro về tài chính kém, khi có cơ hội nâng cao thu nhập họ sẳn sàng bán nông sản với giá cao mà gần như không bị xử phạt gì từ phía doanh nghiệp.

Bảng 6 thể hiện kết quả dự đoán trong mô hình, với kết quả dự đoán đúng là $65,3 \%$. Điều này có nghĩa các hệ số hồi quy trong mô hình là thích hợp cho việc giải thích khả năng tham gia liên kết trong sản xuất khoai tây của nông hộ. Trong số 62 hộ không tham gia thì thời gian tới sẽ có 29 hộ tham gia, trong số 61 hộ có ý định tham gia thì có 16 hộ sẽ tham gia, trong số 122 hộ tham gia thì có 16 hộ sẽ dừng tham gia liên kết.

\section{4. Đề xuất một số giải pháp nhằm nâng cao khả năng tham gia liên kết}

Qua kết quả phân tích thì để nâng cao khả năng tham gia liên kết giữa doanh nghiệp và nông hộ thì cần một số giải pháp như:

Nông hộ và doanh nghiệp cần phải cải thiện các điều kiện thực hiện hợp đồng để mang lại lợi ích nhiều hơn cho các bên trong đó nên áp dụng chính sách giá sàn hay cần áp dụng cách xác định giá trong hợp đồng một cách linh hoạt, vì mục đích cuối cùng của hai bên là nhằm tối đa hoá lợi nhuận (do doanh nghiệp thường chiếm ưu thế về thông tin thị trường nên khi thương lượng về giá cả trong hợp đồng thì nông hộ thường yếu thế hơn). Đồng thời, doanh nghiệp cần xác định giá bán các loại vật tư ứng trước cho nông hộ một cách hợp lý hơn.

Doanh nghiệp cũng cần nâng cao khả năng đánh giá tiềm năng thị trường cũng như tăng cường mở rộng các kênh thị trường trên cơ sở đó cung cấp, chia sẻ thông tin để tạo niềm tin cho nông hộ gắn kết với doanh nghiệp. Mặt khác, doanh nghiệp cần phối hợp với chính quyền địa phương để tăng cường công tác tuyên truyền, nâng cao nhận thức của nông hộ về những lợi ích lâu dài và trách nhiệm trong thực hiện hợp 
Bảng 4. Kết quả ước lượng mô hình hồi quy Logit đa thức

\begin{tabular}{|c|c|c|c|c|}
\hline \multirow{2}{*}{ Diễn giải } & \multicolumn{2}{|c|}{$\mathrm{Y}=2$} & \multicolumn{2}{|c|}{$\mathrm{Y}=2$} \\
\hline & Hệ số & $P^{1}$ & Hệ số & $P^{1}$ \\
\hline $\bar{C}$ & $-6,656$ & & $-1,868$ & \\
\hline $\mathrm{X}_{1}$ (Tuổi chủ hộ) & $-0,024^{\mathrm{ns}}$ & 0,717 & $0,072^{\text {ns }}$ & 0,117 \\
\hline $\mathrm{X}_{2}$ (Trình độ học vấn) & $-0,005^{\mathrm{ns}}$ & 0,371 & $0,002^{\text {ns }}$ & 0,606 \\
\hline $\mathrm{X}_{3}$ (Kinh nghiệm) & $0,525^{*}$ & 0,084 & $0,484^{*}$ & 0,073 \\
\hline $\mathrm{X}_{4}$ (Diện tích) & $1,176^{* * *}$ & 0,007 & $0,513^{*}$ & 0,056 \\
\hline X5 (Lợi nhuận) & $-0,148^{* * *}$ & 0,005 & $-0,069 * * *$ & 0,001 \\
\hline $\mathrm{X}_{6}$ (Chính sách hỗ trợ ) & $1,815^{* * *}$ & 0,003 & $1,966^{* * *}$ & 0,000 \\
\hline $\mathrm{D}_{1}$ (Giới tính) & $-1,740 * *$ & 0,042 & $1,062^{* *}$ & 0,018 \\
\hline \multicolumn{5}{|l|}{ Tồng số: 245} \\
\hline \multicolumn{5}{|l|}{ Pseudo R-Square: 0,2750} \\
\hline \multicolumn{5}{|c|}{ Model fitting information } \\
\hline \multicolumn{5}{|c|}{ Likelihood ration test Chi-square $=140,51 ; \mathrm{DF}=16 ; \mathrm{sig}<0,00000$} \\
\hline
\end{tabular}

Bảng 5. Hệ số tác động biên

\begin{tabular}{lccc}
\hline \multirow{2}{*}{ Diễn giải } & \multicolumn{3}{c}{ Tác động biên } \\
\cline { 2 - 4 } & $\mathrm{Y}=1$ & $\mathrm{Y}=2$ & $\mathrm{Y}=3$ \\
\hline $\mathrm{X}_{1}$ (Tuổi chủ hộ) & $-0,005$ & $-0,009$ & 0,014 \\
$\mathrm{X}_{2}$ (Trình độ học vấn) & 0,001 & $-0,001$ & 0,001 \\
$\mathrm{X}_{3}$ (Kinh nghiệm) & $-0,072$ & 0,030 & 0,041 \\
$\mathrm{X}_{4}$ (Diện tích) & $-0,013$ & 0,196 & 0,183 \\
$\mathrm{X}_{5}$ (Lợi nhuận) & 0,014 & $-0,014$ & $-0,005$ \\
$\mathrm{X}_{6}$ (Chính sách hỗ trợ ) & $-0,278$ & 0,082 & 0,195 \\
$\mathrm{D}_{1}$ (Giới tính) & $-0,008$ & $-0,315$ & 0,323 \\
\hline
\end{tabular}

Bảng 6. Kết quả dự đoán của mô hình

\begin{tabular}{ccccc}
\hline \multirow{2}{*}{ Chỉ tiêu } & \multirow{2}{*}{ Số hộ } & \multicolumn{3}{c}{ Dự đoán của mô hình } \\
\cline { 2 - 5 } & & $\mathrm{Y}=0$ & $\mathrm{Y}=1$ & $\mathrm{Y}=2$ \\
\hline $\mathrm{Y}=0$ & 62 & 33 & 12 & 17 \\
$\mathrm{Y}=1$ & 61 & 7 & 38 & 16 \\
$\mathrm{Y}=2$ & 122 & 16 & 17 & 89 \\
\hline Phần trăm dự đoán đúng & \multicolumn{4}{c}{$65,30 \%$} \\
\hline
\end{tabular}

đồng với doanh nghiệp.

\section{Kết Luận}

Khi tham gia liên kết trong sản xuất khoai tây, nông hộ sẽ dễ tiếp cận với thị trường, với tiê̂n bộ kỹ thuật mới qua việc chuyển giao từ doanh nghiệp. Nghiên cứu đã sử dụng hàm hồi quy Logit đa thức theo phương pháp ước lượng cực đại (MLE) để ước tính khả năng tham gia liên kết trong sản xuất khoai tây của nông hộ. Kết quả ước lượng cho thấy, $14,6 \%$ hộ có khả năng sẽ tham gia liên kết và $63,0 \%$ hộ tham gia liên kết. Bên cạnh đó, kết quả phân tích chỉ ra các yếu tố như kinh nghiệm, diện tích đất nông nghiệp, lợi nhuận, chính sách hỗ trợ và giới tính chủ hộ có ảnh hưởng đến quyết định tham gia liên kết của nông hộ, trong đó biến chính sách hỗ trợ và diện tích có ảnh hưởng mạnh và tích cực nhất đến khả năng tham gia liên kết sản xuất khoai tây của nông hộ. Tuy nhiên, khi giá bán khoai tây tăng thì nông hộ tăng khả năng phá vỡ hợp đồng đã ký kết, do vậy doanh nghiệp cần có chính sách quan tâm hơn nữa đến lợi ích của nông hộ sản xuất khoai tây. 


\section{Tài Liệu Tham Khảo (References)}

Changpetch, P., \& Lin, D. K. J. (2012). Selection of multinomial logit models via association rules analysis. $A d-$ vanced Review 5, 68-77.

Do, G. Q., \& Tran, T. T. (2013). Evaluating probability of joining contract farming of farmer in northern hilly and mountainous region: a case study of tea farmer in Tuyen Quang province. Journal of Agriculture \& Rural Development 11(3), 447-457.

Lam Dong DARD (Deparment of Agriculture and Rural Development of Lam Dong provinve). (2018). Da Lat's potatoes and fake potatoes from China. Retrieved April 9, 2018, from https://tuoitre.vn/khoaitaydatlattuyenchienvoihangtr ungquoc.

MARD (Ministry of Agriculture and Rural Development). (2008) Report No. 578/BC-BNN dated on March 11, 2008. Five-year report of conducting the Decision No. 80/2002/QĐ-TTg of the Prime Minister on policies to encourage the contractual sale of commodity farm produce.

Nguyen, H. D., Tran, T. Q., \& Bui, K. H. T. (2017). Evaluation of factors affecting ability to engage linkage in maize consumption for farmer households in Son La province. Vietnam Journal Agriculture and Science 15(4), 529-536.
Nguyen, T., \& La, K. S. (2014). Research on farmer's economic cooperation need in the Mekong river delta. Journal of Agriculture \& Rural Development 1, 10-16.

Phung, H. G., \& Pham, D. B. (2014). Strengthening the linkages between processing enterprises and shrimp producers in Ca Mau province. Journal of Science and Development 12(2), 231-238.

Tran, N. Q., \& Ikuo, T. (2012). Analyzing causes of failure in contract farming enforcement between farmer and entrepreneur in Vietnam. Journal of Science and Development 10(7), 1069-1077.

Tran, V. M., \& Pham, D. V. (2014). Some solutions for developing contract integration in production - consumption of rice in Dong Thap province. Journal of Science and Development 12(6), 844-852.

Tran, T. Q., Le, C. T. M., Do, G. Q., Bui, D. B., Bui, L. T. M., Nguyen, O. Q., Le, H. T T., Tran, Y. N. T., \& Pham, D. K. (2016). Establishing the process of cooperative production in the use of corn for animal feed. Final Report. Son La, Vietnam: Department of Science and Technology of Son La province.

Tu, T. M. (2016). Solutions to enhance the linking chain of exporting fresh fruits and vegetables for Southern key economic zone. Journal of Science of Ho Chi Minh City Open University 50(5), 123-127. 